\title{
A Customisable And Responsive Design ONLINE BOOKING SYSTEM
}

\author{
Betzabet García-Mendoza and Carlos R. Jaimez-González \\ Departamento de Tecnologías de la Información \\ Universidad Autónoma Metropolitana, Unidad Cuajimalpa, México
}

\begin{abstract}
Internet has become the first option to look for hotels when people travel, which makes it a great opportunity for hotels to have a web site with a booking engine included, which will offer comfort to their clients, and expand their sales to a wider market. This paper presents a customisable online booking system, which facilitates the control of room bookings to administrators of small hotels, managing the sales from their own web site; and facilitates and guarantees the bookings for their potential guests. Additionally, the online system can be automatically visualized in different mobile devices, following the responsive web design pattern.
\end{abstract}

\section{KEYWORDS}

Booking Control System, Customisable Web System, Online Booking System, Responsive Web Design.

\section{INTRODUCTION}

There are several online resources that help planning trips, such as travel agencies, web sites with opinions and comments about hotels and places to visit, social networks, among others. For example, $93 \%$ of the travellers around the world say that their decisions of bookings are influenced by online opinions [1]. When people have decided the city or town to visit, the next step is to look for a place to stay, and given that the first search option is the Internet, it is the best opportunity for hotels to promote their services on it. Currently, there are a great variety of travel agencies, hotel search engines, and other options that allow locating hotels around the world; however, the hotel information shown on those web sites is limited in some cases.

Additionally, $72 \%$ of the travellers around the world state that the possibility of carrying out bookings through a mobile device is very useful; and only $25 \%$ of the hotels allow this functionality [1]. This is the reason why including a booking engine in the web site of a hotel is the best option for those hotels that want to sell in a easy and direct way, offering comfort to their clients, and expanding their sales to a wider market: the Internet.

The main aim of the hotel web sites is to offer rooms, additionally to other general services. These web sites are divided in sections, where they describe the different services offered through text and photo galleries, as well as sections for contacting the hotel, and for requesting room bookings.

This paper presents the analysis, design, implementation and tests of an online booking system, which can be customised by an administrator to set a number of features to manage room bookings. The responsive web design pattern was used to implement the booking system in order to allow its visualization in devices with different resolutions. 
The rest of the paper is organized as follows. Section 2 analyses three existing tools for customisation of hotel web sites. The analysis and design of the system is presented in Section 3, with the structure of web sites for hotels, a study of users, the software methodology used, details of the requirements gathering, and some artefacts generated (use cases, interface prototypes, sequence diagrams and class diagram). Section 4 gives details of the implementation of the system, with a navigation map, a description of the technologies and tools used for the development of the system, the structure of the web portal, and the implementation of the responsive web design pattern. Tests and results are presented in Section 5. Finally, section 6 provides some conclusions and future work.

\section{ExISTING TOOLS}

This section analyses three tools that allow customisation of hotel web sites, which contain an online booking engine: PRO Internacional [2], Creowebs [3] and Obehotel [4].

\subsection{PRO Internacional}

According to its web site, PRO Internacional [2] is a company dedicated to provide integrated web services, which include from the creation of web sites with great amount of functionality and interactivity, up to actions of engagement in social networks and campaigns for positioning a web site on web search engines.

Hotel web sites with an integrated module for online bookings are among the web sites created with PRO Internacional. These web sites have the following functionality: online room bookings, rates by seasons, photo galleries, subscription to an electronic bulletin, comments (optional), surveys, and a mailbox for suggestions. It should be noted that PRO Internacional is not a free tool, and it does not have a demonstration of its functionality.

\subsection{Creowebs}

It is a web tool [3] that allows creating web sites from a variety of default templates, which are designed for different types of web sites, such as blogs, booking systems for hotels or restaurants, among others. Creowebs provides the following modules for the creation of web sites: templates, import content, image editor, blog and news, social networks, videos, music, photo galleries, contact form, payments through Paypal, bookings, use of Google Maps, surveys, visualization of navigation path, version for mobile devices, among others.

A user account was opened in Creowebs in order to explore more about the functionality of this web tool, its advantages and disadvantages. A sample web site for a hotel was created, and different tests were carried out, such as the following: the type of fonts of the web site was changed, the information of certain sections was edited, the forms and templates were modified, some new rooms and seasons were registered with their corresponding prices, some queries to check the availability of rooms were executed, and also some requests for bookings were made. Figure 1 shows the interface for registering a new room in Creowebs.

\subsection{ObeHotel}

According to its creators, ObeHotel [4] is a set of different solutions, developments and technological applications aimed at hotels for marketing and online distribution, oriented to improve the tasks of the hotel administrators, as well as the experience of potential guests. ObeHotel offers four different services: bookings, complete web, channel manager, and maximum visibility. The complete web service offered by ObeHotel was the only service 
analysed, because it is more related to the booking system presented in this paper. The main features of complete web are the following: customisable design, creation and configuration of any type of hotel room, multiple room bookings, management of promotions and packages, positioning of the web site on search web engines, different rates for the web site and for the sale channels, tracking code for monitoring visits of clients, integration with Google Analytics and Google Adwords, and web module for registering client opinions.

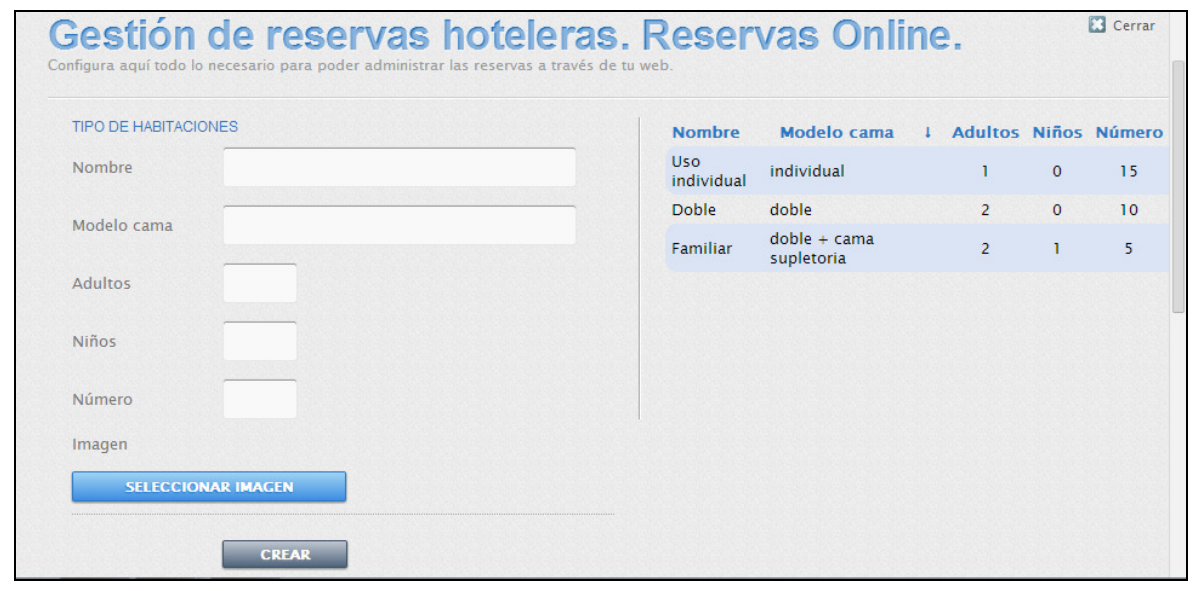

Figure 1. Registration of a new room in Creowebs

ObeHotel provides a demonstration web site, in which users can test the functionality offered, such as the following: verify the availability of rooms; book a room; visualize the different types of rooms; visualize the services, packages and promotions; see a location map of the hotel; fill in a contact form; visualize photo and video tours; among others. Figure 2 shows the user form to verify the availability of rooms.

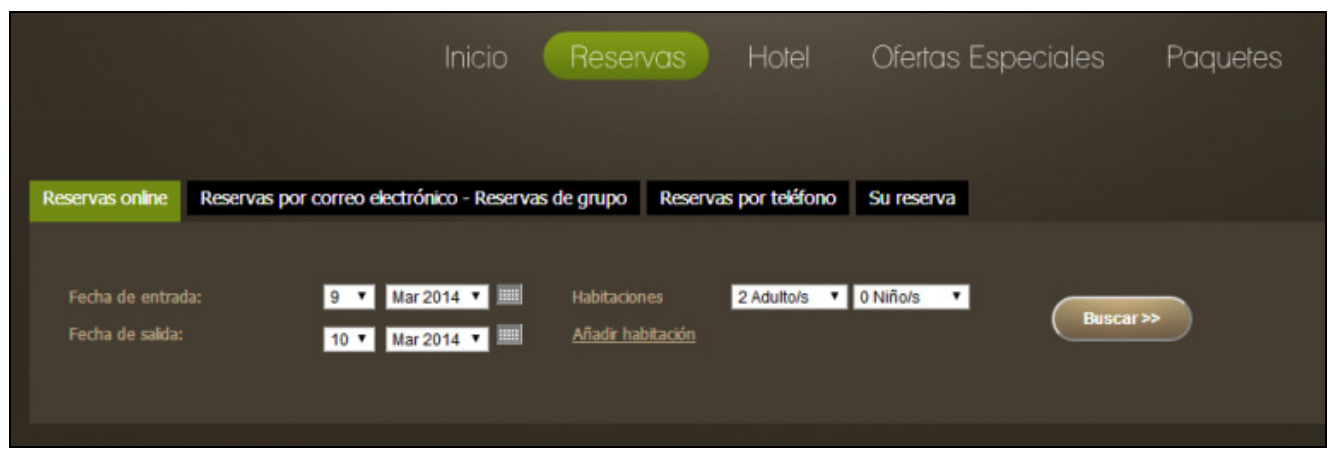

Figure 2. Availability of rooms in ObeHotel

ObeHotel is not a free tool and it does not have a web site for demonstrating the functionality of the administration system. For this reason, it was not possible to test the modules that correspond to the administrator user.

\subsection{Comparison of Tools}

Table 1 shows a comparison of features among the three tools covered in the previous sections: PRO Internacional (TA), Creowebs (TB) and ObeHotel (TC); the tick indicates that the tool has the feature, and the cross indicates that the tool does not have it. It is also provided a brief description of the features shown in Table 1, which were used to evaluate the tools analysed. 
Table 1. Features of tools analysed.

\begin{tabular}{|l|c|c|c|}
\hline \multicolumn{1}{|c|}{ Feature } & TA & TB & TC \\
\hline 1) Management of rooms & $\checkmark$ & $\checkmark$ & $\checkmark$ \\
\hline 2) Multiple bookings & $\mathbf{x}$ & $\mathbf{x}$ & $\checkmark$ \\
\hline 3) Rates by seasons & $\checkmark$ & $\checkmark$ & $\mathbf{x}$ \\
\hline 4) Packages and promotions & $\mathbf{x}$ & $\mathbf{x}$ & $\checkmark$ \\
\hline 5) Availability map & $\checkmark$ & $\checkmark$ & $\checkmark$ \\
\hline 6) Comments & $\checkmark$ & $\checkmark$ & $\checkmark$ \\
\hline 7) Surveys & $\checkmark$ & $\checkmark$ & $\mathbf{x}$ \\
\hline 8) Mailbox for suggestions & $\checkmark$ & $\mathbf{x}$ & $\mathbf{x}$ \\
\hline 9) Default templates & $\checkmark$ & $\checkmark$ & $\mathbf{x}$ \\
\hline 10) Customisable forms & $\mathbf{x}$ & $\checkmark$ & $\mathbf{x}$ \\
\hline 11) Statistics & $\mathbf{x}$ & $\mathbf{x}$ & $\checkmark$ \\
\hline 12) Mobile version & $\mathbf{x}$ & $\checkmark$ & $\mathbf{x}$ \\
\hline
\end{tabular}

The features from Table 1 are the following: 1) Management of rooms allows the administrator to create, update, and delete hotel rooms; 2) Multiple bookings allows multiple bookings of rooms in one transaction, by the same client; 3) Rates by seasons allows to register rates per room according to the season, such seasons are defined by the administrator, with start and end dates, with no possibility of overlaps; 4) Management of packages and promotions allows to create, update, and delete packages and promotions, where promotions are discounts on the price of the services, and packages are a set of services together; 5) Availability map allows to visualize an availability map with the relationship of available and occupied rooms (in some cases it is shown graphically, and in some others it is just a table with all the rooms and their status); 6) Comments allows to include a section for guests to publish comments about their stay at the hotel; 7) Surveys allows the administrator to carry out satisfaction surveys about the stay of the hotel guests; 8) Mailbox for suggestions allows to include a mailbox for suggestions with a form, where the guest can provide a suggestion to the hotel, which will be sent by email to the administrator; 9) Default templates allows the administrator to choose a default template for the design of the web site 10) Customisable forms allows the administrator to customise forms, adding and deleting fields; 11) Statistics allows the administrator to keep track of the visits to the hotel web site; and 12) Mobile version allows to visualize the hotel web site from a mobile device, adjusting the web site according to the resolution of the device.

\section{ANALYSIS AND DESIGN}

This section presents the analysis and design carried out before implementing the online booking system. The structure and information provided in web sites for hotels are described in subsection 3.1 ; these were obtained from an analysis of some web sites. Subsection 3.2 presents the study of users that was carried out, where three user profiles were identified for the system: the administrator profile, the potential guest profile, and the objective hotel profile. The subsection 3.3 describes the software methodology used for the development of the system, which was iterative and incremental. Subsection 3.4 gives details of the requirements gathering. Some use cases are presented in subsection 3.5. The interface model with some interface prototypes designed is presented in subsection 3.6. The subsection 3.7 shows a sequence diagram. Finally, the general class diagram for the system is presented in subsection 3.8. 
International Journal of Computer Science \& Information Technology (IJCSIT) Vol 9, No 5, October 2017

\subsection{Structure and Information of Hotel Web Sites}

Some hotel web sites were visited with the aim of analysing their structure, type of information, and functionality [5]. These hotels are located in touristic places of Mexico [6]: H1) Las cúpulas in Malinalco, Estado de Mexico [7]; H2) La casa del laurel in Taxco, Guerrero [8]; H3) El encanto in Bernal, Querétaro [9]; H4) Hotel Malinalco in Malinalco, Estado de México [10]; H5) Casa abierta in Valle de Bravo, Estado de México [11]; and H6) Hotel Cristal in Chignahuapan, Puebla [12]. Table 2 shows a comparison of the information that is offered in each of the hotel web sites visited; a tick indicates that the hotel has the information or the section in its web site, while a cross indicates that the hotel does not have it. A brief description of the information and sections offered by the hotels analysed is also provided.

Table 2. Information offered on the web sites analysed.

\begin{tabular}{|l|c|c|c|c|c|c|}
\hline \multicolumn{1}{|c|}{ Section } & H1 & H2 & H3 & H4 & H5 & H6 \\
\hline Home/About Who we are & $\checkmark$ & $\checkmark$ & $\checkmark$ & $\checkmark$ & $\checkmark$ & $\checkmark$ \\
\hline Rooms & $\checkmark$ & $\checkmark$ & $\checkmark$ & $\checkmark$ & $\checkmark$ & $\checkmark$ \\
\hline Bookings & $\checkmark$ & $\checkmark$ & $\checkmark$ & x & $\checkmark$ & $\checkmark$ \\
\hline Services & $\checkmark$ & $\checkmark$ & $\checkmark$ & $\checkmark$ & $\checkmark$ & $\checkmark$ \\
\hline Photo gallery & $\checkmark$ & x & x & $\checkmark$ & $\checkmark$ & $\checkmark$ \\
\hline Facilities & $\checkmark$ & x & $\checkmark$ & x & $\checkmark$ & x \\
\hline Packages/Promotions & x & x & x & x & x & $\checkmark$ \\
\hline Rates & $\checkmark$ & $\checkmark$ & $\checkmark$ & x & $\checkmark$ & $\checkmark$ \\
\hline Contact & x & x & $\checkmark$ & $\checkmark$ & $\checkmark$ & $\checkmark$ \\
\hline Location & $\checkmark$ & $\checkmark$ & $\checkmark$ & $\checkmark$ & $\checkmark$ & $\checkmark$ \\
\hline Attractions & $\checkmark$ & $\checkmark$ & $\checkmark$ & $\checkmark$ & x & $\checkmark$ \\
\hline
\end{tabular}

Home/About/Who we are. This section presents a brief description of the hotel, the history of the hotel, the number of years that has been running, the number of rooms, its services, touristic places, among others.

Rooms. This section contains information about the types of rooms that the hotel offers, such as images and description of rooms, furniture used, and services for each room.

Bookings. This section has a form to be filled in by the user with its personal information and the information of the room to be booked. The system notifies the user that the availability of the room will be verified and an email will be sent later with the confirmation of the booking.

Services. This section describes the services provided by the hotel: basic services, such as Internet, parking, cafeteria, among others; and extra services, such as restaurant, bar, business rooms, party rooms, pool, among others.

Gallery. This section provides a photo gallery of the hotel, which can be included in any other section of the web site, or it can have its own separated menu.

Facilities. This section is a combination of the services and gallery sections, it shows the facilities of the hotel through a series of photographs.

Packages/Promotions. This section is composed of packages or promotions defined by the hotel. The packages can contain different extra services added to a room booking; while the promotions can be discounts on the price of the room booking on certain periods of the year. 
Rates. This section shows a table with the prices of each type of room, which depend on the seasons defined by the hotel. The table also shows rates of the extra services provided.

Contact. This section has the contact information of the hotel, such as address, telephone number and email. It can also contain a contact form to be filled in by the user.

Location. This section shows an address and a map with the location of the hotel (Google maps is used in some cases).

Attractions. The attractions section contains information of the main touristic attractions. In some cases it also has information about touristic packages.

The main aim of the system presented in this paper is to reach small hotels located in touristic places in Mexico, which currently lack of information systems for controlling their bookings.

\subsection{User Profiles}

User profiles describe features of the system users, and they allow to design a system centered on the needs of the users; this way it is obtained a system with a better level of usability. The study of users carried out consisted on identifying the users of the booking system, designing a survey for each type of user with questions that allowed obtaining information about their interests, needs and features, which were taken into consideration for the design of the system.

There were two users identified: administrator and potential guest. There was also a hotel profile, that defines the specific features for the hotels to which the system is aimed at. A total of 16 surveys were carried out to administrators of hotels located in the magic tows of Huasca de Ocampo and Real del Monte, both towns belong to the Hidalgo state in Mexico. There were also a total of 84 surveys to tourists that were found in the magic towns previously mentioned.

The survey carried out on the hotel administrators was based on obtaining information about several aspects, such as the following: how long the hotel has been running, number of rooms in the hotel, total capacity of the hotel, existing web page for the hotel, methods used for booking control, ways to make bookings on the hotel, way of booking considered to be more comfortable and efficient, how the hotel promotes its services, where its guests come from, and places that its guests visit during their stay. The survey carried out on the potential guests was based on obtaining information about their ways of searching hotels, their preferences to choose a hotel, and the ways used to book a hotel room.

Once the surveys were carried out, they were analysed and with the results obtained, it was possible to define the user profiles, which are described in the following paragraphs. It should be noted that the profiles shown in this paper were defined according to the features found in the majority of the people that answered the surveys.

Administrator profile. This profile has the following features: he or she is a person between 35 and 60 years old, born in the magic town or touristic place, and digital literate. The administrator profile refers to the hotel administrator, which is the person in charge of administering all the functionality of the hotel web site.

Potential guest profile. This profile has the following features: he or she is a person between 25 and 40 years old, digital literate and has studied an undergraduate or postgraduate degree. The potential guest is used to going out of his/her place of residence when having free time, which could be on weekends, public holidays or holidays. He or she stays at the places that visit, likes 
visiting touristic attractions, emblematic buildings, historical monuments, as well as places rich in handicrafts and gastronomy. The potential guest carries out the search for hotels through the Internet, books currently by using the telephone and email; although, if possible he/she would prefer booking through the hotel web site if it existed.

Objective hotel profile. This profile has the following features: it is located in a magic town or in a place where there is a touristic attraction or other attractions such as emblematic buildings, handicraft production, cultural parties, traditions, among others. Due to these factors the flow of tourists is considerable. The hotel identified by this profile has been operating between 1 and 10 years, it is relatively small, it has approximately 15 rooms with a total capacity of 40 people. The hotel controls its bookings through a registration book; the bookings can be made by telephone, email or in person at the reception. The hotel has a computer and Internet service at the reception. The hotel has no web page, and it promotes its services through the city council web pages, in which its name, contact telephones and address are published. Other ways of promoting its services are the following: magazines, leaflets, presentation cards, and recommendations by other guests; considering the last one as the one that gives best results.

\subsection{Software Methodology}

An iterative and incremental methodology was used for the development of this project, as shown in Figure 3, which consists on carrying out several iterations with the following stages in each of them: analysis, design, implementation and testing $[13,14]$. This methodology is incremental because there is new functionality added to the system in each iteration.

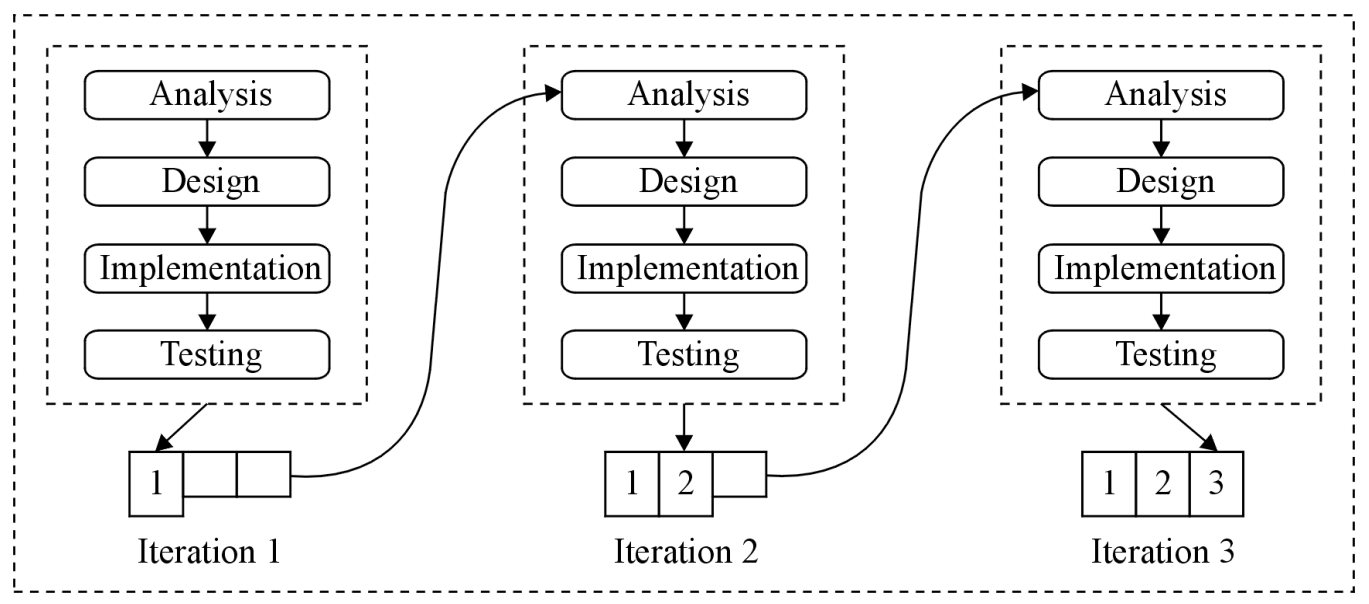

Figure 3. Iterative and incremental software methodology

With this methodology it is possible to have a functional prototype since the first iteration, and evaluate with the client such prototype to get feedback and solve problems in early stages of the project. Using an iterative and incremental approach allows to have a more stable version of the system at the end of each iteration, which has more quality and new functionality compared with previous versions.

The booking system took four iterations in order to be finished, in which new modules were added to the system up to complete the whole functionality. Some modifications were carried out according to the feedback received by the client, and also corrections were made after some errors were detected in the test stages from previous iterations. 


\subsection{Requirements Gathering}

The requirements for the booking system were gathered from Bosque Magico hotel, which was one of the hotels that answered the survey, and whose administrator agreed to provide all the information needed. This hotel fulfilled with all the features defined on the profiles described previously. It should be noted that there were four hotel administrators that agreed to provide information for the system, but the Bosque Magico hotel was selected because of the variety of options and services that offers, which allowed to have a more general design of the system. The requirements obtained from the interviews with the hotel administrator were the following.

It was required a web site that: 1) shows the types of rooms and their features; 2) shows the packages and services offered; 3 ) shows the rates of each room by season; 4) shows the touristic attractions that can be visited during the stay at the hotel, as well as other interesting activities; 5) allows to book one or more rooms; 6) allows to book a package.

It was required a module that allows the administrator to: 1) define the types of rooms and their features; 2) define seasons with their corresponding start and end dates; 3) define the prices of the different types of rooms, according to the registered seasons; 4) define packages with the rooms and services included; 5) define services offered by the hotel, and their features; 6) visualize the status of each of the rooms (available/occupied), and in case of being occupied to be able to see the information of the user that booked it; 7) get a list of the users that have stayed at the hotel, with their basic information and email.

It was required a module that allows to make bookings through the hotel web site, following the next steps: 1) the user will need to provide check-in date, check-out date and number of people to stay; 2) the system will verify the availability of the hotel for that criteria, and will display a list of available rooms; 3) the user will select the room or rooms wanted and input his/her personal information, such as full name, address, telephone number and email; 4) the system will show the booking information and ask the user to confirm it; 5) the system will send an email to the user with the booking information.

\subsection{Use Cases}

Uses cases were used to specify the behaviour of the system [15], which show the interaction of the system with the actors (administrator and potential guest). Figure 4 shows the general use case diagram, where both actors interact with the different modules of the system.

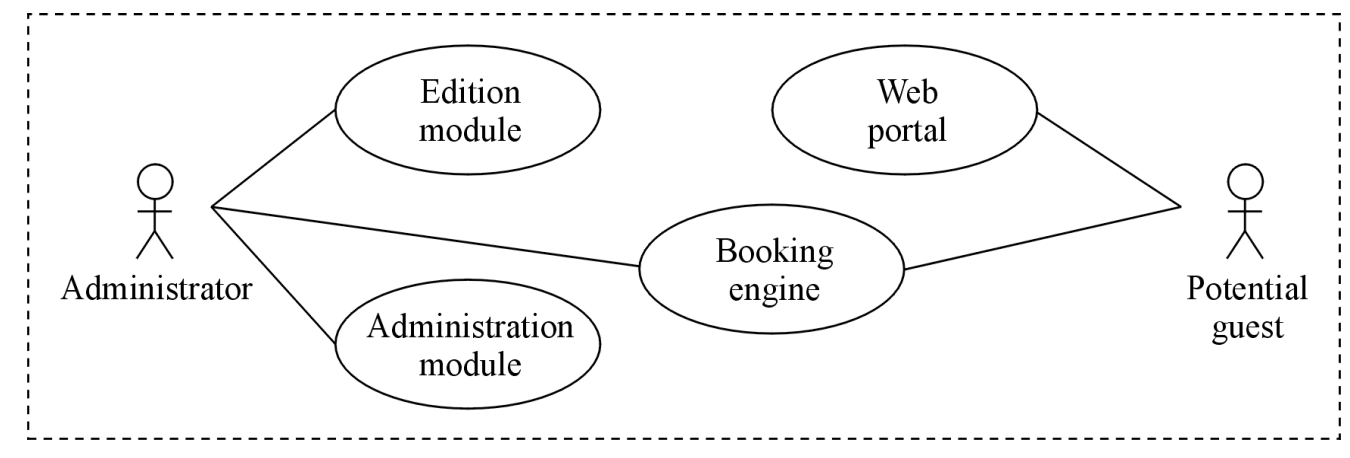

Figure 4. General use case diagram

The booking system is composed by four main modules: edition, administration, web portal, and booking engine, which are briefly described in the following paragraphs. 
Edition module. It allows the administrator to customise the web portal with the features of the hotel. The administrator provides information to the system about rooms, packages, services, bookings, among others.

Administration module. It allows the administrator to manage the bookings of the hotel. The administrator is able to visualize, through a calendar, the availability of the hotel. Additionally, the administrator can access the information of each booking.

Booking engine. This module allows the potential guest and the administrator to verify the availability of the hotel by providing check-in date, check-out date, and number of people. They are also able to book one or more of the rooms shown on the availability list.

Web portal. It allows the potential guest to navigate through the web portal that was customised by the administrator through the edition module. In this web portal the potential guest can make bookings and find information about packages, rooms, services, attractions, among others.

Given that the booking is the most important transaction of the system, in the following subsections it will be used as example to show some of the artefacts generated. The first step to make a booking in the system is to verify the availability of the hotel. In order to do that, the system will request the user to provide check-in date, check-out date, and number of people to stay. This information allows the system to make a specific search in the database, and return an availability room list with the selected dates to the potential guest. In the next step, the potential guest will select the room or rooms that want to book. After that, the potential guest will input his/her personal information, such as first name, last names, email, contact telephone number, address, and others. Once this is carried out, the system will display the information of the booking with the check-in date, the check-out date, type and number of rooms, total price of the booking, and it will request the potential guest to confirm the booking. Finally, the potential guest will confirm the booking, and the system will send an email to the registered account of the potential guest. Figure 5 shows the Booking use case diagram, where this transaction requires two actions or use cases: Verify availability and Book. It should be noted that administrator and potential guest interact with this use case normally.

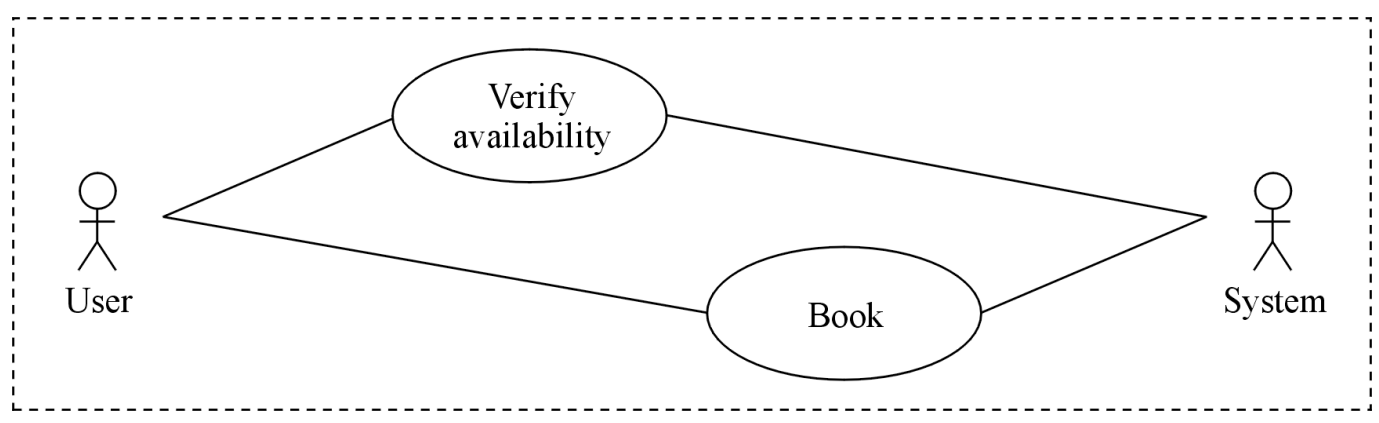

Figure 5. Booking use case diagram

\subsection{Interface Model}

This subsection shows an interface prototype designed for the booking module, which was created in the Balsamiq Mockups online tool. Figure 6 shows the Bookings section of the web portal, which is visualized after the potential guest has clicked on the Bookings menu. In this prototype the potential guest provides check-in date, check-out date, and number of people. 
Taking into consideration the use case described in the previous subsection, there are three more interface prototypes to complete the actions of that use case: 1) one that shows a list of the rooms that fulfil the request of the potential guest, where a room or rooms can be selected;2) one that shows a web form to be filled by the potential guest with personal information, such as name, address, telephone number and email; and 3) one that results from the selection of the room to book, in which the interface shows the complete information of the booking and the personal information provided by the potential guest.

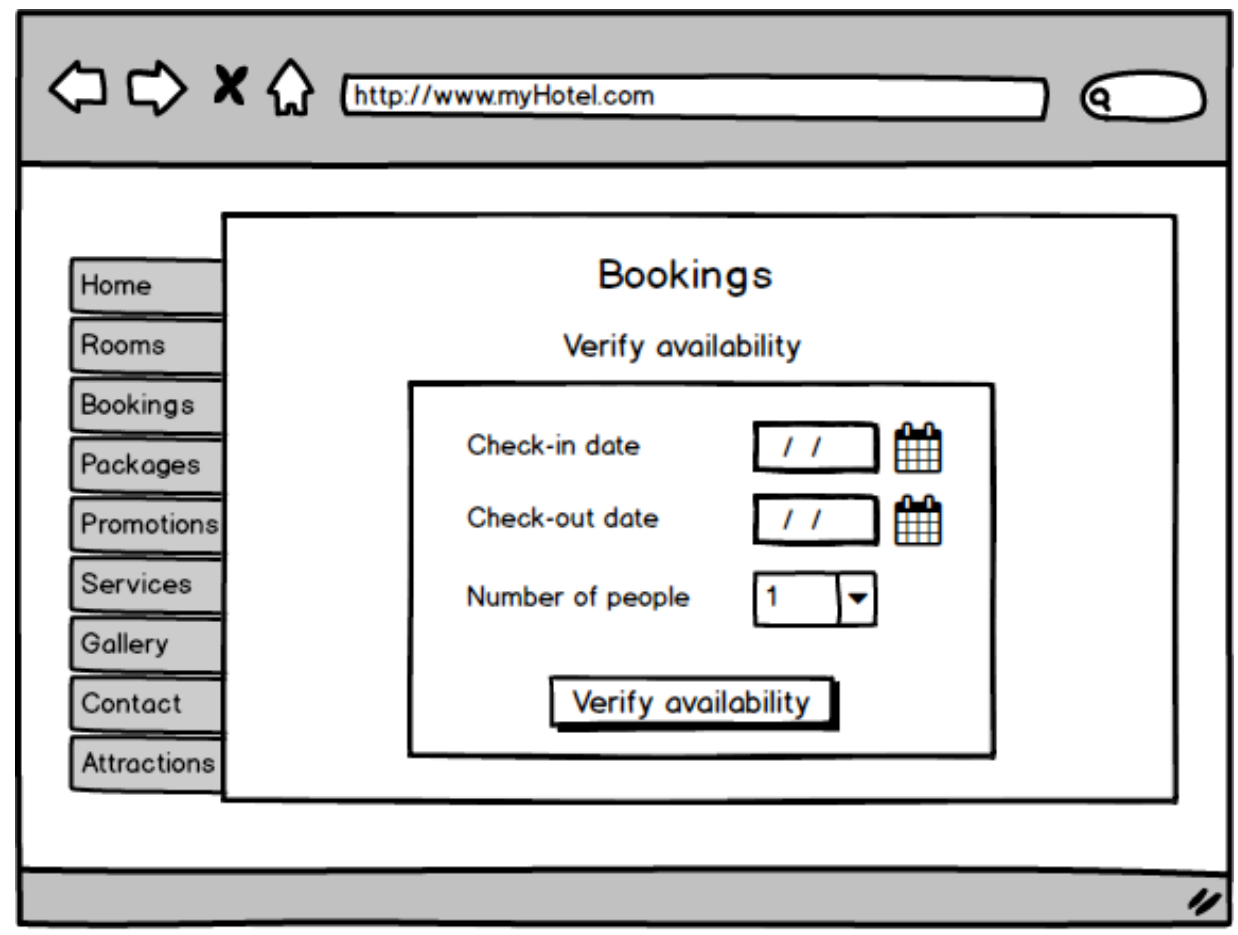

Figure 6. Interface prototype for the Booking section

\subsection{Sequence Diagrams}

This subsection presents only a sequence diagram that corresponds to one of the use cases presented in a previous subsection of this paper: Verify availability, which is shown in Figure 7.

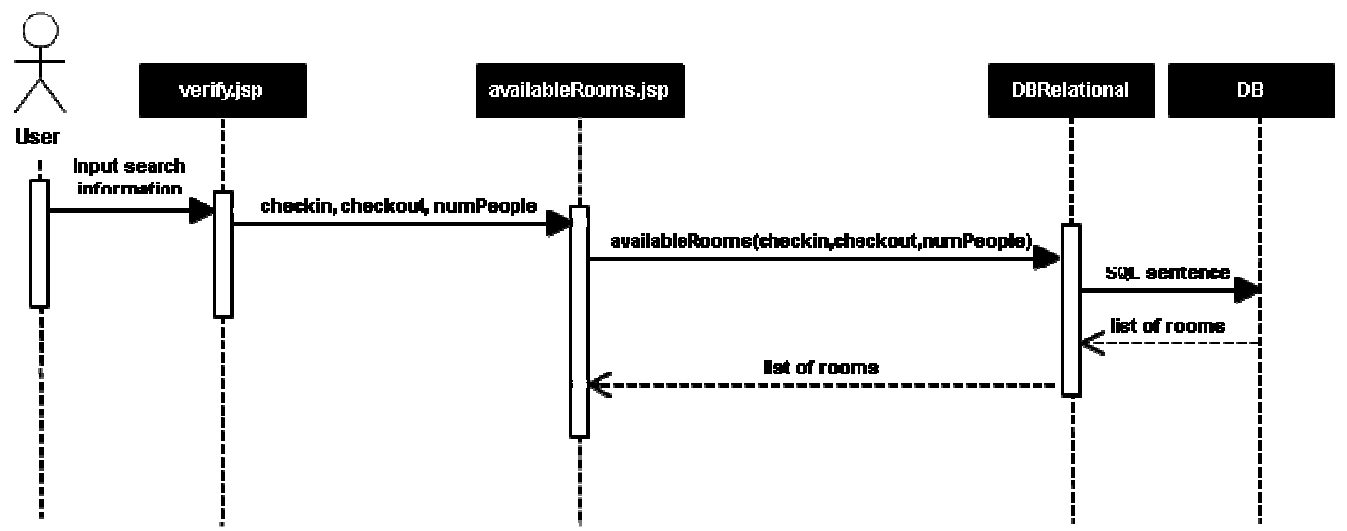

Figure 7. Sequence diagram to verify availability of rooms 
International Journal of Computer Science \& Information Technology (IJCSIT) Vol 9, No 5, October 2017

\subsection{Class Diagram}

This subsection presents the class diagram for the booking system, which is shown in Figure 8. Some of the classes included are the following: Usuario (User), Habitacion (Room), Paquete (Package), Temporada (Season), Reservacion (Booking), Cliente (Client), Servicio (Service), Promocion (Promotion), among others. Each class is presented along with its attributes.

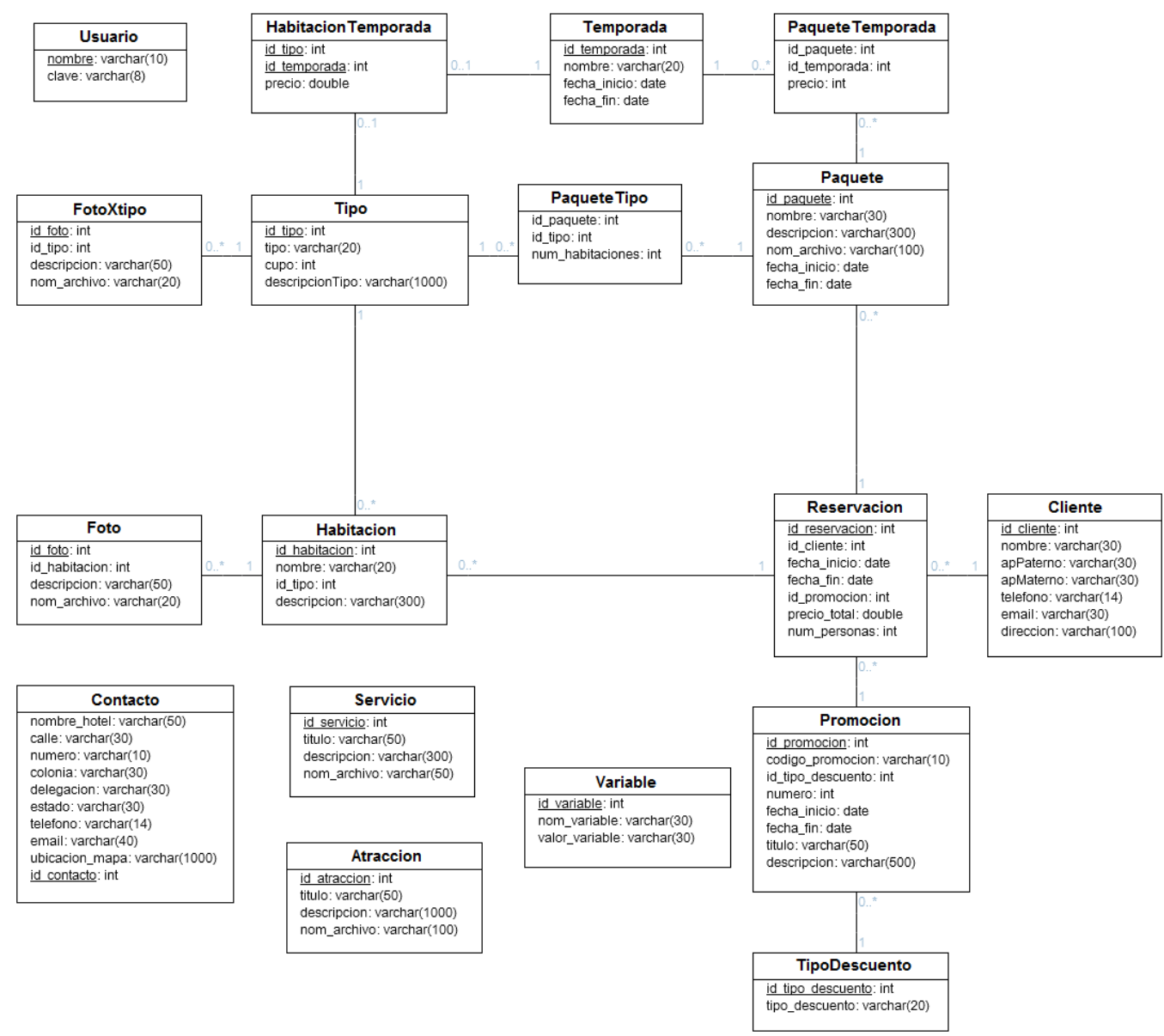

Figure 8. Class diagram for the booking system

\section{IMPLEMENTATION OF THE SYSTEM}

The implementation of the booking system is presented in this section. Subsection 4.1 shows the navigation map of the system, with the two users of the system: hotel administrator, and potential guest. Subsection 4.2 describes the technologies and tools used for the development of the system. The structure of the web portal is presented in subsection 4.3. Finally, subsection 4.4 describes the implementation of the responsive web design pattern, which allowed to adjust the interface of the web portal to different devices, in order to improve the visualization and navigation experience. A preliminary implementation of the system was presented in [16]. 


\subsection{Navigation Map}

Figure 9 shows the navigation map of the system, with the two users of the system: hotel administrator and potential guest. The navigation map shows the set of web pages to which each user has access.

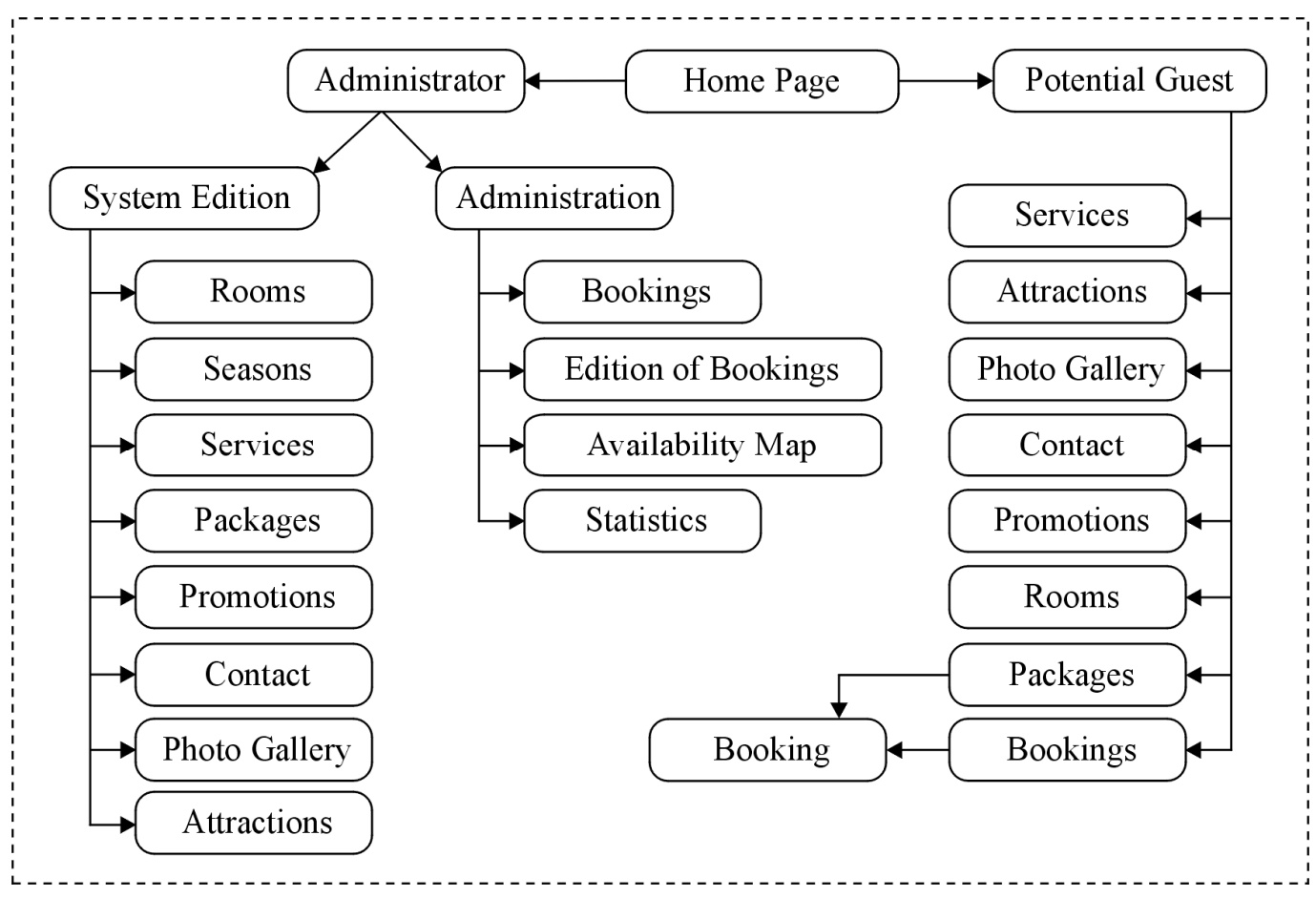

Figure 9. Navigation map

The administrator can access two sections mainly: system edition and administration. In the system edition, the administrator can customise the web portal by creating, modifying and deleting the following elements: rooms, seasons, services, packages, promotions, contact information, photo galleries, and attractions. In the administration section, the administrator can manage all the bookings, make bookings manually in the system, modify existing bookings, visualize bookings on the availability map of rooms, and display statistics of the system.

The potential guest has access to all the web pages customised by the administrator: services, attractions, photo galleries, contact information, promotions, rooms, packages, and booking of rooms and packages. In order to make a booking of a room or package, the potential guest can verify the availability of rooms in the booking web page.

\subsection{Technologies and Tools Used}

There were several technologies and tools used for the development of the system presented in this paper. Some of them are described in the following paragraphs.

Java Server Pages (JSP). It is a Java technology that allows to develop dynamic web pages, combining static HTML with HTML dynamically generated. JSP files were used for the creation of dynamic web pages, where information is input to the system, extracted from the database and processed. They were also used to create different objects dynamically, validate users, among other operations. 
JavaScript. It is an interpreted programming language, designed to make interactive and dynamic web pages. This language is based on objects because it has an implementation of the Document Object Model (DOM), which is a model that translates the structure of an HTML document to a tree of objects when the document is interpreted by a web browser. Every object has methods and attributes that can be invoked from JavaScript code, so that any element in an HTML document can be modified. JavaScript can be embedded directly in the HTML document or by using separate files, which can be executed when required. JavaScript was used for the creation of dynamic web pages, specially for the dynamic structure of the information to be displayed on the different devices in which the booking system is visualized: laptop, tablet and smart phone.

Cascading Style Sheets (CSS). It is a language for styles used to present information in a web page. Through CSS it is possible to change everything related to the presentation of a web page, such as the background colour, font family, font size, font colour, text align, border size, border type, border colour, margins, padding, position of elements, among others. The mechanism to change the position of HTML elements in a web page is widely used for organizing information when accessing a web portal from mobile devices, because the visualization space available in each device can be different. For this reason, CSS version 3 includes Media Queries, which allow to know the size of the device screen (or web browser window) from which the web page has been requested, and based on this result it is possible to apply different blocks of styles for different sizes of devices. CSS and Media Queries were used for the presentation of the web portal interface and for the correct identification of the different device sizes, which allowed to apply different styles.

NetBeans. It is an Integrated Development Environment (IDE), which allows to write, compile, debug and execute programs. NetBeans allowed to concentrate the creation of all the files of the project, such as Java classes, JSP files, and JavaScript files.

Apache Tomcat. It is a web server and servlet container, which allows to compile JSP files that are converted into servlets. Apache Tomcat was used as a web server, servlet container, and for processing all the JSP files of the system.

MySQL. It is a Relational Database Management System (RDBMS) based on the Structured Query Language (SQL), multi-threaded, multi-user and multi-platform, which is widely used for storing data in web applications for different platforms. MySQL was used for the creation of the relational database of the system; for executing Data Definition Language (DDL) sentences, such as create, alter and drop; and for executing Data Manipulation Language (DML) sentences, such as select for making queries, insert for adding registries, update for modifying registries, and delete for eliminating registries from a table.

Balsamiq Mockups. It is an application for creating prototypes for web graphical interfaces, with the aim of showing them to the clients of the project in early stages of the development process. This tool allows the designer to make diagrams with built-in designs, using a What You See Is What You Get (WYSIWYG) graphic editor by dragging and dropping elements on a work area. This application allows to represent all the elements used for the implementation of a web page, such as browser windows, titles, headers, menus, images, videos, among others. Balsamiq Mockups was used to create interface prototypes very quickly, which were used to validate and test the interface with the hotel administrator.

\subsection{Structure of the Web Portal}

This section provides details of the web portal structure, which is shown in Figure 10. A brief description of the contents of each directory that compose the web portal is also provided in the following paragraphs. 


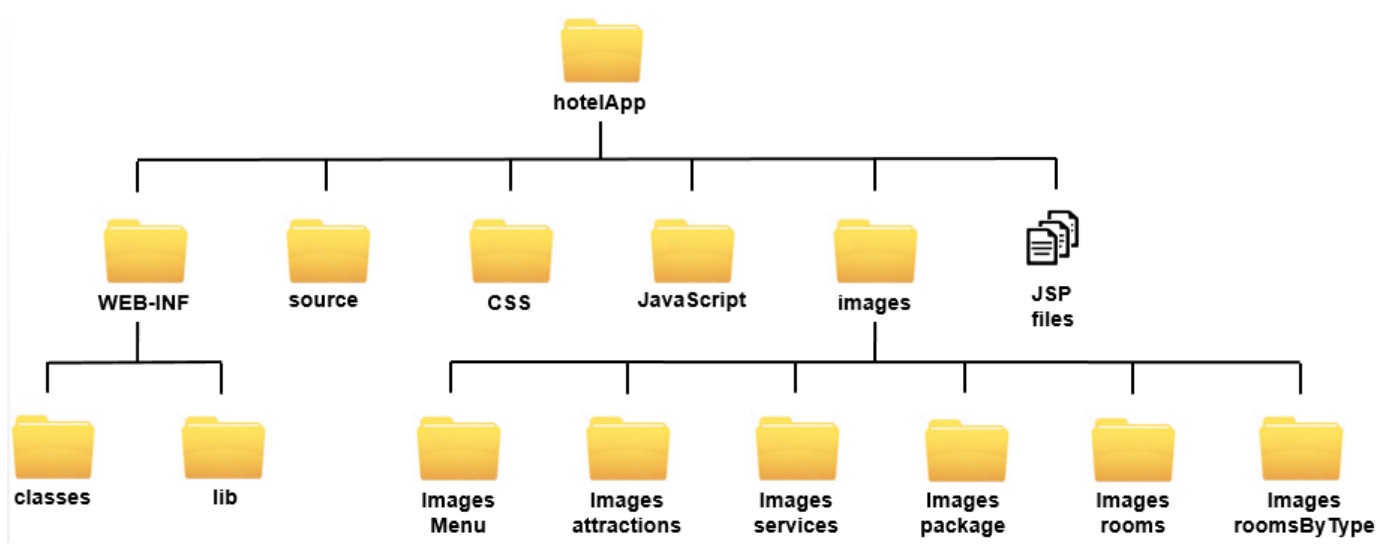

Figure 10. Structure of the web portal

WEB-INF directory. It has two directories inside: the classes directory contains the class files used by the system, which are the result of compiling the .java files of the project; and the lib directory contains the .jar files used by the project, which are libraries that provide some functionality for the system, such as database connectivity, upload of files to the web server, email management, among others.

Source directory. It contains all the .java files, which correspond to the Java classes used by the system. These files are organized in packages.

CSS directory. It has .css files used for the styles and presentation of the web portal. These files contain some Media Queries for applying different blocks of styles for different resolutions of devices.

JavaScript directory. It contains all the .js files, which define some functions to make interactive and dynamic web pages. They are also used for organizing dynamically the information on the web portal according to the device, and for validating user forms.

Images directory. It has five directories inside: the imagesAttractions directory contains images uploaded by the hotel administrator for the touristic attractions section of the web portal; the imagesServices directory has images uploaded by the hotel administrator for the services section of the web portal; the imagesPackage directory contains images uploaded by the hotel administrator for the packages section of the web portal; the imagesRooms directory has images uploaded by the hotel administrator for each of the rooms created in the editing section of the web portal; and the imagesRoomsByType directory has images uploaded by the hotel administrator for each of the types of rooms created in the editing section of the web portal.

JSP files. The root directory of the web portal contains all the .jsp files, which are used for the dynamic web pages used in the system. Inside each JSP file there is a combination of HTML and scriptlets JSP, as well as links to CSS, JavaScript and JQuery files.

\subsection{Responsive Web Design}

Responsive Web Design (RWD) [17,18,19] is a design pattern that allows to adapt the interface of a web portal to different devices in order to improve the visualization and navigation experience of users. Currently, there are a great variety of mobile devices with different resolutions and screen sizes, this is the main reason why the design of web interfaces requires to 
adapt to these devices. Some examples of web and e-commerce applications that use RWD can be found in $[20,21,22,23]$.

RWD is based on media queries, which are a series of instructions that define certain features of width, height, orientation, colour and other parameters of the device from which the web portal is accessed. This allows to apply different styles for each device by determining its screen size or resolution, so it is possible to have different views of the same web portal: a view for a mobile phone, a view for a tablet, a view for a desktop computer, etc.

The booking system presented in this paper used three media queries for the different devices that access the web portal, which are shown in Figure 11.

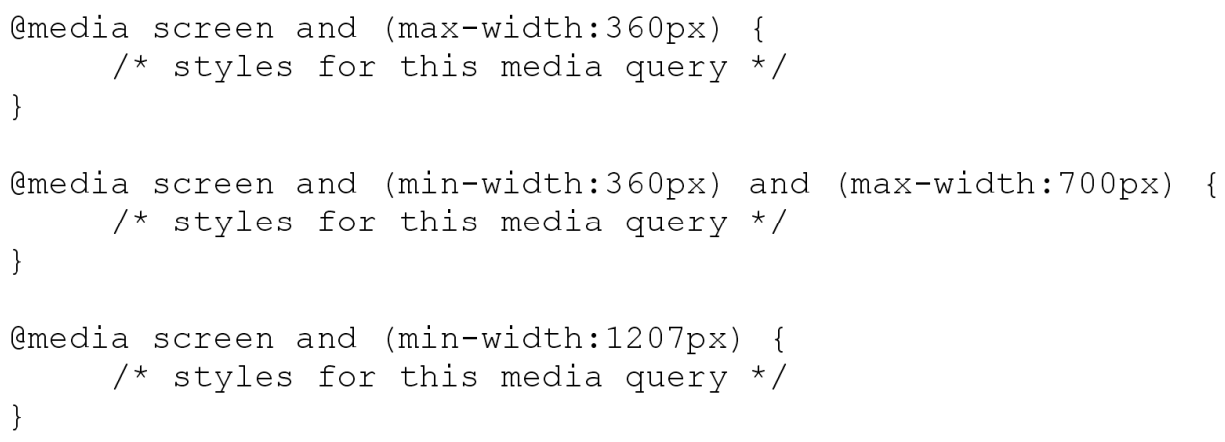

Figure 11. Media queries used by the booking system

The first media query defines a section of styles that will be applied to the web portal when it is accessed from a device with a maximum CSS width of 360 pixels, which defines the view of a mobile phone. The second media query defines a segment of styles that will be applied to the web portal when it is accessed from a device with a minimum CSS width of 360 pixels and a maximum CSS width of 700 pixels, which defines the view of a tablet. The third media query defines a fragment of styles that will be applied to the web portal when it is accessed from a device with a minimum CSS width of 1207 pixels, which defines the view of a laptop or desktop computer. The screen word indicates that the resolution of the web browser window will be taken into consideration instead of the resolution of the device. The resolution of the web browser window is also known as the CSS width, which was the one used for the booking system, because of the great difference in resolutions (density of pixels per inch) in mobile devices with the same size of screen (in inches).

In some cases media queries are not flexible for organizing information. This was the case of the booking system, in which certain information is created dynamically, and the amount of elements to be visualized on the web portal is variable. For this reason, in this system it was necessary the implementation of several JavaScript functions, which allowed the organization of information according to the device from which the web portal was accessed. The JavaScript functions created were controlled using the window.innerWidth and the window.innerHeight attributes, which allowed to know the size of the web browser window, so it could be determined the number of columns to be used to divide the information on the web page for visualization. The JavaScript functions created are executed when the onresize and the onload events of the web browser are fired.

Because currently there are different devices from which a web site can be accessed, three views can be defined for its visualization: a view for mobile phones, a view for tablets, and a view for laptops and desktop computers. The most frequent way of distributing information is through a specific number of columns according to the view; for example: one column for the view for 
mobile phones, two columns for the view for tablets, and three columns for the view for laptops and desktop computers. Figure 12 shows an example of how the distribution in columns can be arranged according to the device: mobile phone, tablet, and laptop or desktop computer.

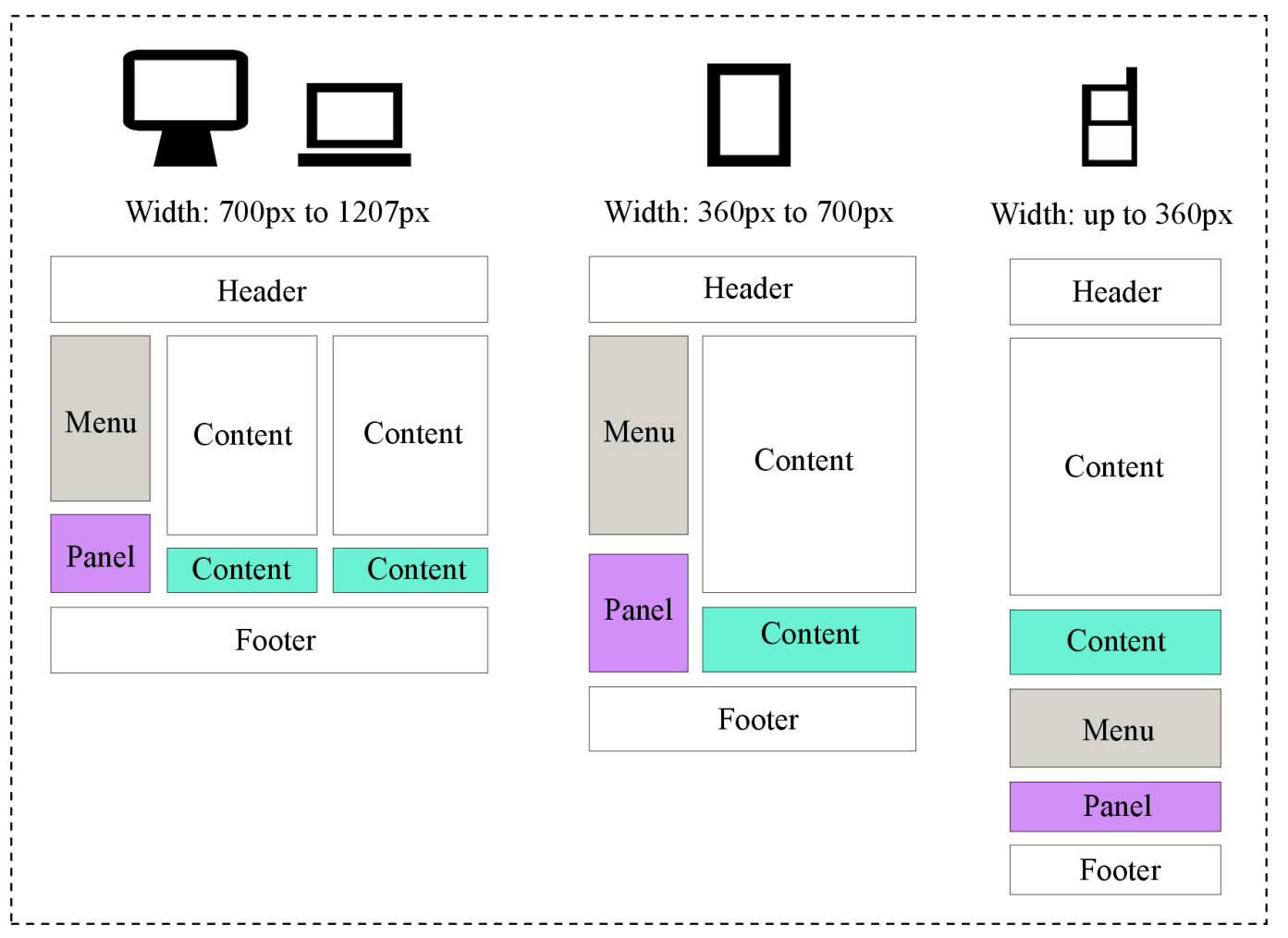

Figure 12. Distribution of information in columns

\section{TeStS AND Results}

This section presents the tests carried out on the system and their results. There were two types of tests: functionality tests and interface tests.

The functionality tests were carried out through test controls to determine the success or failure of the system on specific tasks, which were part of the specification gathered from the user, and written as the use cases found in the analysis and design stages of the software methodology.

The results of these tests were as follows: $92 \%$ of the test controls were successful, while $8 \%$ of them failed. It should be noted that failed tests concerned with tasks where the dates of bookings overlapped, and with some prices that were out of range according to the season.

The interface tests were carried out to check the correct visualization of the web portal on the different devices for which it was designed: mobile phone, tablet, laptop and desktop computer. Table 3 shows the devices on which the web portal was tested. The columns of the table indicate the following information about the device: brand, model, operating system, web browser, and resolution of the screen. It is also shown the screenshots of the visualization of the web portal on three different devices from Table 3: the laptop \#1, the tablet \#3 and the mobile phone \#5. The screenshots provided show a web page with the rooms available in a hotel, in three different resolutions, according to the device where the web site is being displayed. 
Table 3. Devices used in the interface tests.

\begin{tabular}{|c|c|c|c|c|c|c|}
\hline Device & $\#$ & Brand & Model & O. S. & Browser & Resolution \\
\hline Laptop & 1 & Dell & XPS - M1330 & Windows & Chrome & $1280 \mathrm{px}$ by 800px \\
\cline { 2 - 7 } & 2 & HP & Pavilion 15p213ns & Windows & Chrome & $1366 \mathrm{px}$ by 768px \\
\hline \multirow{2}{*}{ Tablet } & 3 & Apple & iPad mini 2 & iOS 8 & Safari & $768 \mathrm{px}$ by 1024px \\
\cline { 2 - 7 } & 4 & Samsung & Galaxy Tab 4 & Android & Chrome & $800 \mathrm{px}$ by 1280px \\
\hline \multirow{2}{*}{ Phone } & 5 & Alcatel & One touch & Android & Android & $320 \mathrm{px}$ by 568px \\
\cline { 2 - 7 } & 6 & Samsung & Galaxy S4 & Android & Android & $360 \mathrm{px}$ by 640px \\
\hline
\end{tabular}

Figure 13 shows the web interface displayed on a desktop computer with a resolution of 1240 by 1024 pixels, which is the maximum; Figure 14 shows the web interface displayed on a tablet with a resolution of 720 by 960 pixels; and Figure 15 shows the same web interface displayed on a smart phone with a resolution of 320 by 480 pixels.

The web page in Figure 13 is divided in two panels: the top panel, which has the menu with the different options for the potential guest; and the bottom panel, which is actually the panel for displaying the contents of the page, and contains the rooms available organized in three columns.

The screenshot in Figure 14 shows the same web page, but with reduced information and simplified menus, due to the resolution of a tablet. The information of rooms available is organized in two columns, and the menu is hidden from the top, but it will appear on the left if the user clicks on the icon located on the right top corner of the web page.

Figure 15 shows the same web page, but displayed on a mobile phone. It can be noted that the rooms available are organized in one single column, and the menu is also hidden, but it will appear on the left if the user clicks on the icon located on the right top corner of the web page.

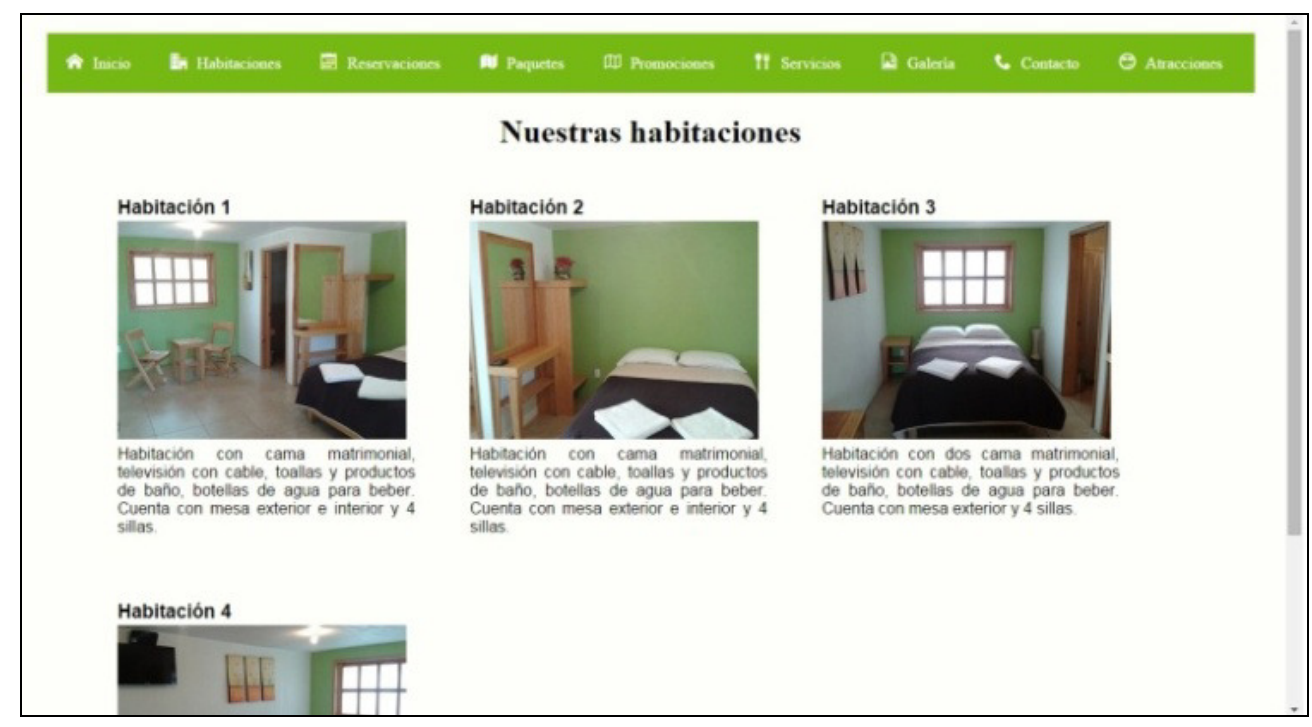

Figure 13. System displayed on a desktop computer (1240px by 1024px) 
International Journal of Computer Science \& Information Technology (IJCSIT) Vol 9, No 5, October 2017

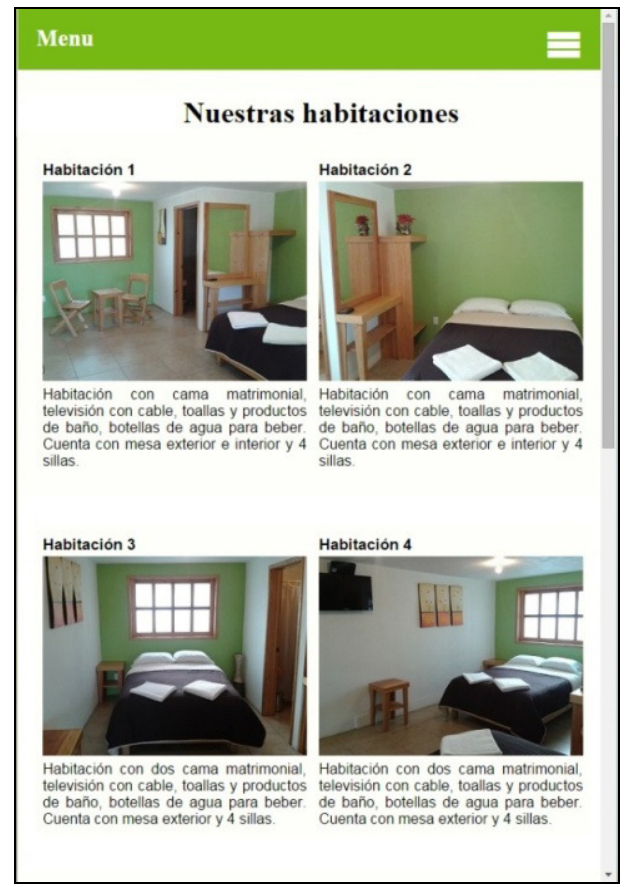

Figure 14. System displayed on a tablet (720px by 960px)

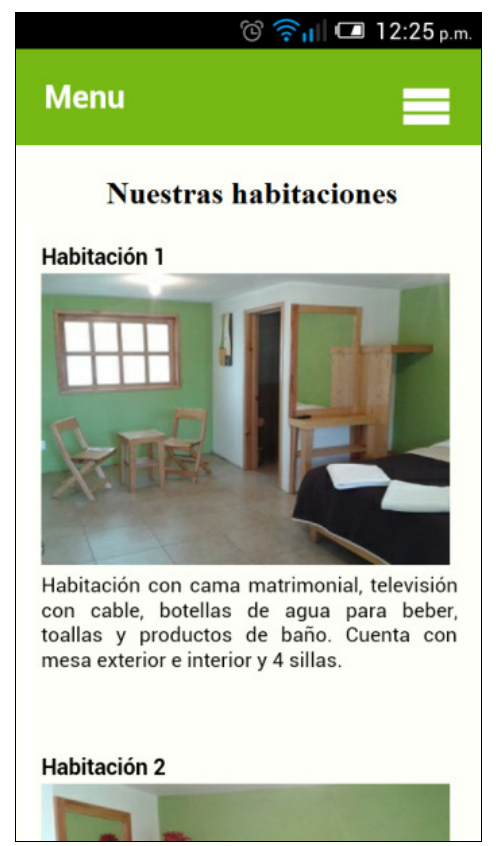

Figure 15. System displayed on a mobile phone (320px by 480px) 


\section{Conclusions And Future Work}

This paper presented a customisable online booking system to control room bookings. In order to allow the system to be visualized in different mobile devices, it was implemented using the responsive web design pattern.

An analysis and a comparison of similar existing tools were also presented. The analysis and design of the system were described, along with a study of users, the software methodology used, details of the requirements gathering, and some artefacts generated. Details of the implementation of the system were also given, with its navigation map, a description of the technologies and tools used for the development of the system, the structure of the web portal, and the implementation of the responsive web design pattern.

Functionality and interface tests were described, where it was shown that the system functionality was tested using test controls, while the interface was tested on different laptops, desktop computers, tablets and mobile phones. It was shown that the system successfully adapted itself to all the different devices used. Some screenshots of the interfaces displayed on three devices were presented: on a desktop computer with a resolution of 1240 by 1024 pixels, on a tablet with a resolution of 720 by 960 pixels, and on a mobile phone with a resolution of 320 by 480 pixels.

Further work is needed to carry out usability tests with hotel administrators, as well as with potential guests. Finally, the system will be fine-tuned after the tests, and it will be put into production in the hotels for which it was designed.

\section{REFERENCES}

[1] Trip Barometer: Survey carried out to hotel owners and travellers, http://www.tecnohotelnews.com/wp-content/uploads/2013/03/Infografia_TripBarometer.jpg, last access in August 2017.

[2] Web site of PRO Internacional, http://www.prointernacional.com/, last access in August 2017.

[3] Web site of Creowebs, https://creowebs.com/, last access in August 2017.

[4] Web site of ObeHotel, http://www.obehotel.com/, last access in August 2017.

[5] García-Mendoza, B., Jaimez-González, C. R. (2014). Propuesta de Sistema Web Personalizable para el Control de Reservaciones en Hoteles. Journal of Research in Computing Science. 79, 135145 .

[6] Bellota, L. A. (2017). Programa de Pueblos Mágicos: valorización turística de la cultura. México: Centro de Estudios Sociales y de Opinión Pública.

[7] Web site of the hotel Las cúpulas, http://lascupulas.com.mx/. last access in August 2017.

[8] Web site of the hotel La casa del laurel, http://www.hotelentaxco.com/es/index.html, last access in August 2017.

[9] Web site of the hotel El encanto, http://elencantobernal.com/acerca, last access in August 2017.

[10] Web site of the hotel Hotel Malinalco, http://www.hotelmalinalco.com.mx/, last access in August 2017.

[11] Web site of the hotel Casa abierta, http://www.casabierta.com.mx/index.html, last access in August 2017.

[12] Web site of the hotel Hotel Cristal, http://www.hotelcristalchignahuapan.com.mx/, last access in August 2017.

[13] Jacobson, I., Booch, G., Rumbaugh, J. (1999). The Unified Software Development Process. Boston, Massachusetts, USA: Addison-Wesley Professional.

[14] Booch, G., Rumbaugh, J., Jacobson, I. (2005). The Unified Modelling Language User Guide. Boston, Massachusetts, USA: Addison-Wesley Professional.

[15] Sommerville, I. (2015). Software Engineering. Essex, England: Pearson Education Limited.

[16] García-Mendoza, B., Jaimez-González, C. R. (2016). Sistema Web Personalizable con Diseño Web Adaptable para la Administración de Reservaciones en Hoteles. Journal of Research in Computing Science. 125, 75-86. 
[17] Frain, B. (2015). Responsive Web Design with HTML5 and CSS3. Birmingham, England: Packt Publishing.

[18] Zea, R. (2015). Mastering Responsive Web Design. Birmingham, England: Packt Publishing.

[19] Marcotte, M. (2011). Responsive Web Design. New York, USA: A Book Apart.

[20] Zeng, Y., Gao, J., Wu, C. (2014). Responsive Web Design and Its Use by an E-Commerce Website. In: Rau P.L.P. (Eds.). Cross-Cultural Design. CCD 2014. Lecture Notes in Computer Science, vol. 8528. Springer.

[21] Jaimez-González, C. R., Ortíz-González, R. (2014). A Configurable Web System for Generating E-Commerce Web Sites. Journal of Research in Computing Science: Advances in Computing Science, 81, 93-102.

[22] Bernacki, J., Blazejczyk, I., Indyka-Piasecka, A., Kopel, M., Kukla, E., Trawiński, B. (2016). Responsive Web Design: Testing Usability of Mobile Web Applications. In: Nguyen N.T., Trawinski B., Fujita H., Hong T.P. (Eds.). Intelligent Information and Database Systems. ACIIDS 2016. Lecture Notes in Computer Science, vol. 9621. Springer, Berlin, Heidelberg.

[23] Nebeling, M., Norrie, M. C. (2013). Responsive Design and Development: Methods, Technologies and Current Issues. In: Daniel F., Dolog P., Li Q. (Eds.). Web Engineering. ICWE 2013. Lecture Notes in Computer Science, vol. 7977. Springer, Berlin, Heidelberg.

\section{Authors}

Betzabet García-Mendoza is currently studying a Master degree in Computer Science, Design and Communication at the Universidad Autónoma Metropolitana Campus Cuajimalpa, in Mexico City. She received her Bachelor degree in Information Technologies and Systems from the same university in 2016. Her research interests include Technologies for Supporting Education, and the Development of Web and E-Commerce Applications.

Carlos R. Jaimez-González is an Associate Professor at the Information Technologies Department at the Universidad Autónoma Metropolitana Campus Cuajimalpa, in Mexico City. He received his $\mathrm{PhD}$ degree in Computer Science at the University of Essex, United Kingdom in 2011. His research interests include Technologies for Supporting Education, Interoperability in Distributed Systems, XML and Related Technologies, and the Development of Web and E-Commerce Applications. He has a distinction as a National Researcher from the Mexican Government.
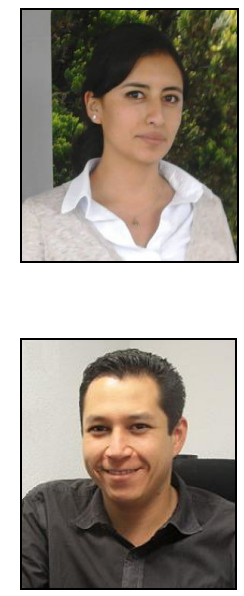